\title{
Navigating the Fas lane to improved cellular therapy for cancer
}

\author{
Madhav V. Dhodapkar \\ Winship Cancer Institute, Emory University, Atlanta, Ceorgia, USA.
}

Genetically engineered T cells have shown promising activity in the

treatment of cancer. However, these cells are also potentially susceptible to immune-suppressive pathways in the tumor microenvironment that may limit their efficacy. In this issue of the $J C l$, Yamamoto et al. describe a new cellular engineering approach to prevent Fas-mediated inhibition of $\mathrm{T}$ cell function, which may be exploited to improve cellular therapy for cancer.

\section{Efficacy of adoptively} transferred T cells hindered by lack of persistence

Adoptive cellular therapies (ACTs) based on $\mathrm{T}$ cell redirection via chimeric antigen receptors (CARs) have led to remarkable tumor regressions in some hematologic cancers, leading to approvals for therapy in pediatric acute lymphoblastic leukemia and adult aggressive B cell lymphoma (1-3). Promising results have also been achieved with $\mathrm{T}$ cells engineered to express $\mathrm{T}$ cell receptors against tumor antigens or $\mathrm{T}$ cells isolated from tumors. Yet many patients receiving these therapies experience recurrent disease. Novel approaches to not only improve the efficacy of these therapies but also extend their application to solid tumors are highly desired. Longterm persistence of functional $\mathrm{T}$ cells following adoptive transfer has emerged as a consistent correlate for clinical efficacy of ACT $(2,4)$. Therefore, strategies that lead to enhanced persistence of adoptively transferred T cells are an important translational goal for improving ACT.

Several factors impact the efficacy of adoptive cell therapies; these include variables relating to the cell therapy product (e.g., type of T cell, nature of costimulatory domains), tumor target (e.g., expression of target antigen), or immune-suppressive factors/signals in the tumor microenvironment $(2,3)$. These considerations have led to several approaches to improve ACT, particularly CAR-based redirection therapies, including utilizing central memory or stem cell memory T cells (5), or T cells with defined ratios of CD4 to CD8 (6) as substrates; modifications in costimulatory domains (7); as well as strategies for dual antigen targeting.

Clinical protocols for ACT currently rely on a phase of ex vivo $\mathrm{T}$ cell expansion to generate adequate numbers of cells (typically $10^{8}-10^{10}$ cells) needed for treatment. This process leads to progressive alterations in epigenetic markers (8), gene expression, and metabolic profiles (9), leading eventually to a heterogenous pool of $\mathrm{T}$ cells that differ in their capability to persist, expand, and mediate tumor regression following adoptive transfer. Despite this heterogeneity, certain markers such as Fas, a member of the TNF receptor family, are commonly expressed on most cells utilized for ACT and therefore may be a broadly applicable target.

\section{Role of Fas in T cell death}

Interaction of Fas with Fas ligand (FasL) represents a physiologic pathway to downregulate immune responses through the induction of apoptosis in activated Fas

\section{Related Article: p. 1551}

Conflict of interest: MVD serves in an advisory role with Genentech, Amgen, Roche, Janssen, Kite, and Lava Therapeutics. Copyright: (๑) 2019 American Society for Clinical Investigation

Reference information: / Clin Invest. 2019;129(4):1522-1523. https://doi.org/10.1172/JCI127581.

$\mathrm{T}$ cells during immune response (10). As FasL seems to be commonly expressed in the tumor milieu, this may be an important obstacle in the persistence and efficacy of ACT. In this issue, Yamamoto and colleagues show, in a series of elegant experiments, that $\mathrm{T}$ cells engineered to express Fas variants with impaired capacity for downstream signaling act as dominant negative receptors (DNRs), preventing FasL-mediated apoptosis of T cells (ref. 11 and Figure 1). T cells co-engineered with a Fas DNR and either a tumor-specific T cell receptor or CAR led to improved antitumor activity in more than one model system. As the authors suggest, this approach may therefore provide a strategy to improve antitumor ACT across multiple platforms and antigenic targets.

The role of Fas in death/survival of $\mathrm{T}$ cells is well established. Recent studies point to non-apoptotic effects of Fasmediated signaling on $\mathrm{T}$ cells, including $\mathrm{T}$ cell costimulation and promoting terminal differentiation of $\mathrm{CD} 4^{+}$and $\mathrm{CD} 8^{+}$ $\mathrm{T}$ cells (12). Loss-of-function mutations in Fas have been detected in patients with autoimmune lymphoproliferation syndrome (ALPS), which leads to expansion of aberrant double-negative $\mathrm{T}$ cells (13). Effects of $\mathrm{T}$ cell-specific deletion of Fas signaling and the development of aberrant lymphoproliferation and autoimmune phenotype in mice seem to be strain specific, suggesting that yetundetermined factors may influence the risk for these phenotypes after inhibition of Fas signaling in T cells (14). T cell-specific ablation of Fas in C57BL/6 mice led to aberrant lymphoid homeostasis and the development of lung disease resembling idiopathic pulmonary fibrosis over time (14). Infusion of Fas DNR-engineered cells did not seem to lead to such phenotypes in the context of the models studied in the current article. Nonetheless, the complexity and straindependent effects observed with alteration of Fas signaling in murine $\mathrm{T}$ cells, 
A

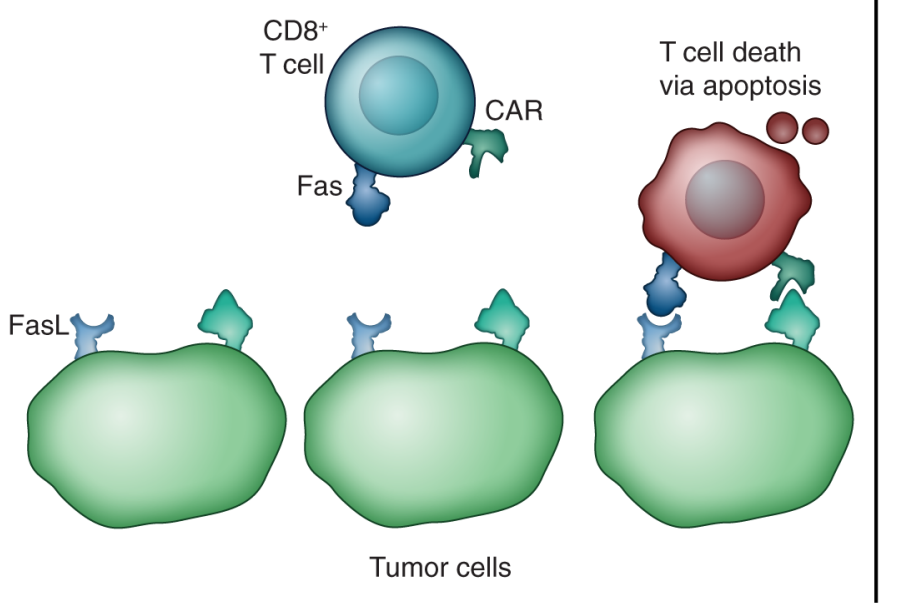

B

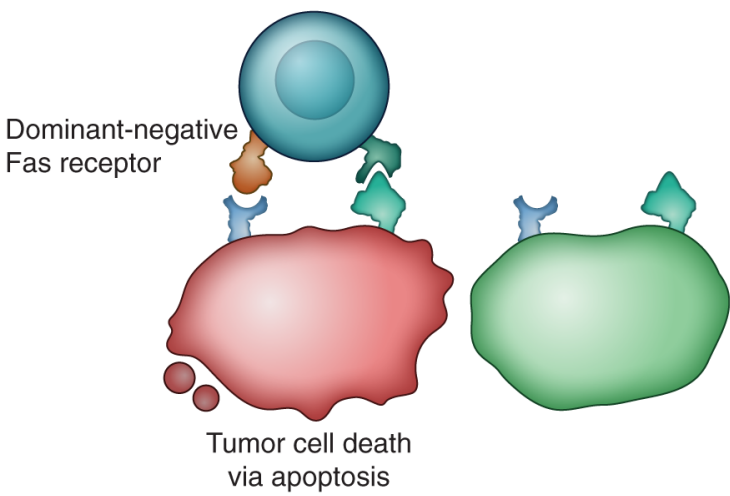

Figure 1. Schematic representation of Fas signaling in tumor cells during CAR T cell therapy. (A) FasL-mediated inhibition of adoptive cell transfer. The apoptosis-inducing ligand FasL is preferentially expressed on tumor cells. T cells used for adoptive immunotherapy constitutively express Fas, the receptor for FasL. Cognate Fas-FasL interaction limits T cell persistence and ultimately affects antitumor efficacy. (B) Overcoming FasL-mediated inhibition. Genetically engineered dominant-negative Fas variants impair Fas-FasL binding and prevent FasL-induced apoptosis. This leads to longer persistence of T cells and to better tumor regression and enhanced survival. Figure illustrated by Rachel Davidowitz.

and prior studies with detection of Fas mutations in patients with ALPS (13) and $\mathrm{T}$ cell lymphoma $(15,16)$, suggest that potential toxicity signals and risk of aberrant lymphoproliferation or malignancy will need to be carefully monitored as this approach is translated to the clinic.

\section{Enhancing persistence of adoptively transferred T cells}

Several other approaches are also being pursued to overcome the inhibitory signals or cells in the tumor microenvironment. These include therapeutic combinations with drugs or antibodies targeting these cells/factors or genetic engineering of $\mathrm{T}$ cells to impart resistance to inhibition in the tumor bed $(17,18)$. Fas DNR can now be added as another strategy to achieve this goal. This may be particularly useful in the setting of tumors that overexpress FasL, which appears to be the case across diverse tumor types.

In summary, the studies by Yamamoto and colleagues describe preclinical evidence supporting a novel approach to enhance ACT efficacy across a broad range of human tumors. We await successful translation of this strategy for testing in the clinic to improve ACT. Strategies to improve persistence of genetically modified $\mathrm{T}$ cells following transfer will go a long way in building on the initial successes with ACT.

\section{Acknowledgments}

MVD is supported in part by funds from the NIH (R35 CA197603), Multiple Myeloma Research Foundation, Leukemia and Lymphoma Society, and International Waldenstrom's Macroglobulinemia Foundation.

Address correspondence to: Madhav Dhodapkar, Winship Cancer Institute, 1760 Haygood Drive, Atlanta, Georgia 30322, USA. Phone: 404.778.1900; Email: madhav.v.dhodapkar@emory.edu.

1. Boyiadzis MM, et al. Chimeric antigen receptor (CAR) T therapies for the treatment of hematologic malignancies: clinical perspective and significance. J Immunother Cancer. 2018;6(1):137.

2. Sadelain M, Riviere I, Riddell S. Therapeutic T cell engineering. Nature. 2017;545(7655):423-431.

3. Guedan S, Ruella M, June CH. Emerging cellular therapies for cancer [published online ahead of print December 10, 2018]. Annu Rev Immunol. https://doi.org/10.1146/annurev-immunol042718-041407.

4. Neelapu SS, et al. Axicabtagene ciloleucel CAR $\mathrm{T}$-cell therapy in refractory large B-cell lymphoma. N Engl J Med. 2017;377(26):2531-2544.

5. Gattinoni L, Klebanoff CA, Restifo NP. Pharmacologic induction of $\mathrm{CD}^{+} \mathrm{T}$ cell memory: better living through chemistry. Sci Transl Med. 2009;1(11):11ps12.

6. Turtle CJ, et al. CD19 CAR-T cells of defined $\mathrm{CD}^{+}: \mathrm{CD}^{+}$composition in adult B cell ALL patients. J Clin Invest. 2016;126(6):2123-2138.

7. Guedan S, et al. Enhancing CAR T cell persistence through ICOS and 4-1BB costimulation. JCI Insight. 2018;3(1):e96976.

8. Henning AN, Roychoudhuri R, Restifo NP. Epigenetic control of CD8(+) T cell differentia- tion. Nat Rev Immunol. 2018;18(5):340-356.

9. Kishton RJ, Sukumar M, Restifo NP. Metabolic regulation of $\mathrm{T}$ cell longevity and function in tumor immunotherapy. Cell Metab. 2017;26(1):94-109.

10. Strasser A, Jost PJ, Nagata S. The many roles of FAS receptor signaling in the immune system. Immunity. 2009;30(2):180-192.

11. Yamamoto TN, et al. T cells genetically engineered to overcome death signaling enhance adoptive cancer immunotherapy. J Clin Invest. 2019;129(4):1551-1565.

12. Cruz AC, et al. Fas/CD95 prevents autoimmunity independently of lipid raft localization and efficient apoptosis induction. Nat Commun. 2016;7:13895

13. Fisher GH, et al. Dominant interfering Fas gene mutations impair apoptosis in a human autoimmune lymphoproliferative syndrome. Cell. 1995;81(6):935-946.

14. Hao Z, Hampel B, Yagita H, Rajewsky K. T cellspecific ablation of Fas leads to Fas ligandmediated lymphocyte depletion and inflammatory pulmonary fibrosis. J Exp Med. 2004;199(10):1355-1365.

15. Takakuwa T, et al. Frequent mutations of Fas gene in nasal NK/T cell lymphoma. Oncogene. 2002;21(30):4702-4705.

16. Takakuwa T, Dong Z, Takayama H, Matsuzuka F, Nagata S, Aozasa K. Frequent mutations of Fas gene in thyroid lymphoma. Cancer Res. 2001;61(4):1382-1385.

17. Cherkassky L, et al. Human CAR T cells with cell-intrinsic PD-1 checkpoint blockade resist tumor-mediated inhibition. JClin Invest. 2016;126(8):3130-3144.

18. Bollard CM, et al. Tumor-specific T-cells engineered to overcome tumor immune evasion induce clinical responses in patients with relapsed Hodgkin lymphoma. J Clin Oncol. 2018;36(11):1128-1139. 